\title{
Contrast Enhancement in Transform Domain
}

\author{
Gwanggil Jeon \\ Department of Embedded Systems Engineering, Incheon National University \\ 119 Academy-ro, Yeonsu-gu, Incheon 406-772, Korea \\ gjeon@inu.ac.kr
}

\begin{abstract}
The contrast enhancement is an important research field in image processing. The histogram equalization stretches the dynamic range of intensity and histogram equalization results contrast enhanced image. In this paper, we apply contrast limited adaptive histogram equalization in certain band (low-low) of wavelet transform. Then, we apply low-low area normalization to balance image brightness. Simulation result section provides performance comparison. Three metrics were used for assessing objective performance. In addition, visual performance comparison is provided.
\end{abstract}

Keywords: Image processing, contrast enhancement, wavelet transform, low-low band, performance assessment

\section{Introduction}

The goal of the image processing is to raise low contrast and decrease the unwanted artifacts. The image contrast enhancement methods have been researched principally on grayscale and frequency domain. The histogram equalization (HE) is one of the most actively researched spatial domain method which is used to increase the image contrast [1-5]. The expectation of $\mathrm{HE}$ is to generate better quality image from noisy or lowcontrast image. It is known that the reason of unpleasant area is large peaks. To remove this artifacts, one can use HE to increase the boring area and remove noisy peaks. As this work is contrast enhancement, we only focus on contrast enhancement issue, and leave denoising issue.

The contrast enhancement can be fulfilled in wavelet transform domain. The main difference between the Fourier transform and the wavelet transform is the later can analyze non-stationary signal. In addition, the wavelet transform provides promising results for localization in both of spatial and frequency domain. Therefore, wavelet transform is now well used in image processing such as image enlargement, enhancement, compression, and denoising [6-8].

The conventional HE method is to create a new map of input image. Thus, the histogram of output image is resulted as uniformly distributed. Figure 1 shows an example of original image and its conventional HE. Although the histogram is stretched, Figure 1(a) looks more natural. The large peaks of the histogram brought comparatively similar area such as flat background. Moreover, result image could become totally dark or bright [9-16]. In this paper, a new method is proposed which outperforms conventional HE. The remainder of this paper is arranged as follow. Section 2 provides brief introduction of wavelet transform and the proposed contract enhancement approach. Section 3 provides simulation results with three objective performance metrics and visual performance comparison. Finally, Section 5 yields conclusion remarks. 


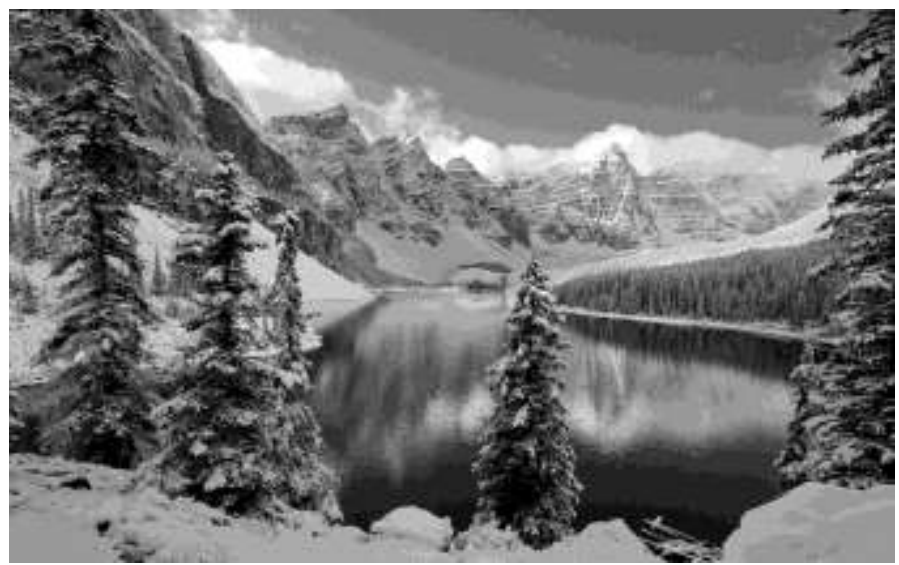

(a)

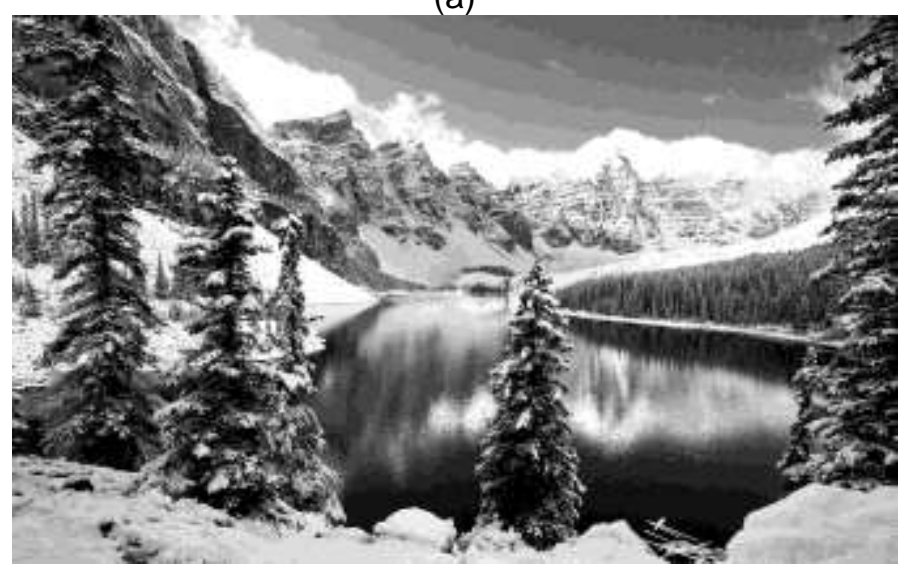

(b)

Figure 1. Image Comparison: (a) Original Image, (b) Result of Conventional Histogram Equalization

\section{Proposed Method}

In this paper, we improve contrast limited adaptive histogram equalization method proposed in [8]. The contrast limited adaptive histogram equalization is operated by block-based processing. There are two configurable parameters in this approach: the first one is block side and the other one is clip limit. By modifying two parameters, one can control image quality. The image can be represented as a 2D array of coefficients, and each coefficient stands for the intensity in each position. In general, an image can be represented by two signals, the low frequency variation and the high frequency variation. The low frequency variation forms the basis of an image, and the high frequency variation represents details.

In the discrete wavelet transform, the wavelets are discretely acquired. The wavelet transform can be used in several image processing areas and they include image denoising, image matching, image segmentation, image restoration and compression. Recently, wavelet transform tool is widely used in medial imaging. There are several wavelets such as Haar wavelet, Daubechies wavelet, and the dual-tree complex wavelet transform. There are scaling function, $\varphi(t)$, and wavelet function, $\psi(t)$, in wavelet transform. For example, the Haar wavelet's mother wavelet function is, 


$$
\psi(t)=\left\{\begin{array}{lc}
1 & 0 \leq t<1 / 2, \\
-1 & 1 / 2 \leq t<1, \\
0 & \text { Otherwise. }
\end{array}\right.
$$

Here, the scaling function is shown as,

$$
\phi(t)= \begin{cases}1 & 0 \leq t<1, \\ 0 & \text { otherwise. }\end{cases}
$$

Figure 2 shows two level discrete wavelet transform of the 'Cameraman' image.

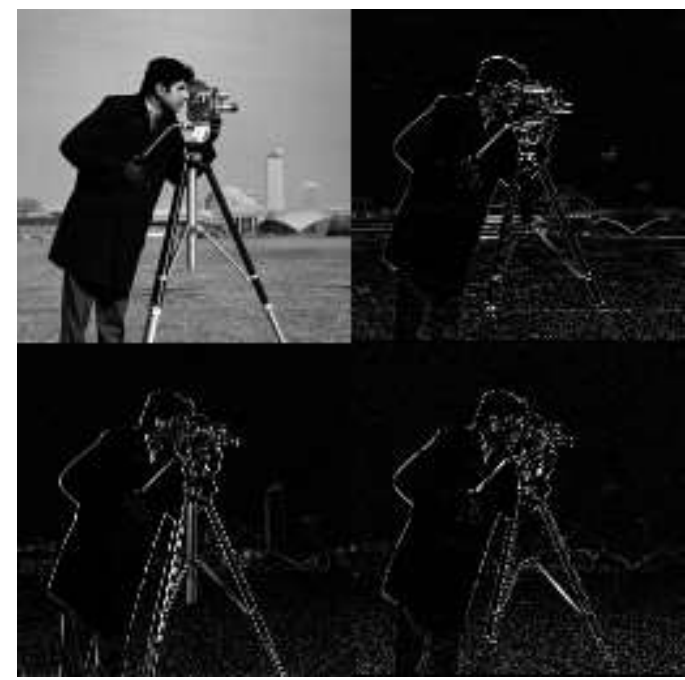

(a)

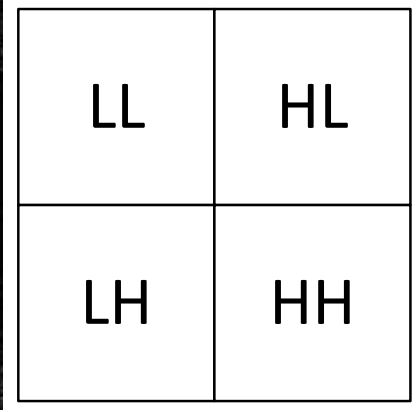

(b)

\section{Figure 2. Two Level Discrete Wavelet Transform of the 'Cameraman' Image}

Figure 3 shows the block diagram of the proposed method. In this paper, we used 'Haar' wavelet as shown in Eqs. (1) and (2). The resulting two-dimensional array of coefficients contains four bands of data, each represented as low-low (LL), high-low (HL), low-high (LH), and high-high (HH). The LL band can be decomposed more times in the similar way and generate more subbands. We apply contrast limited adaptive histogram equalization method in LL suband. After that, we apply LL area normalization to balance image intensity. 


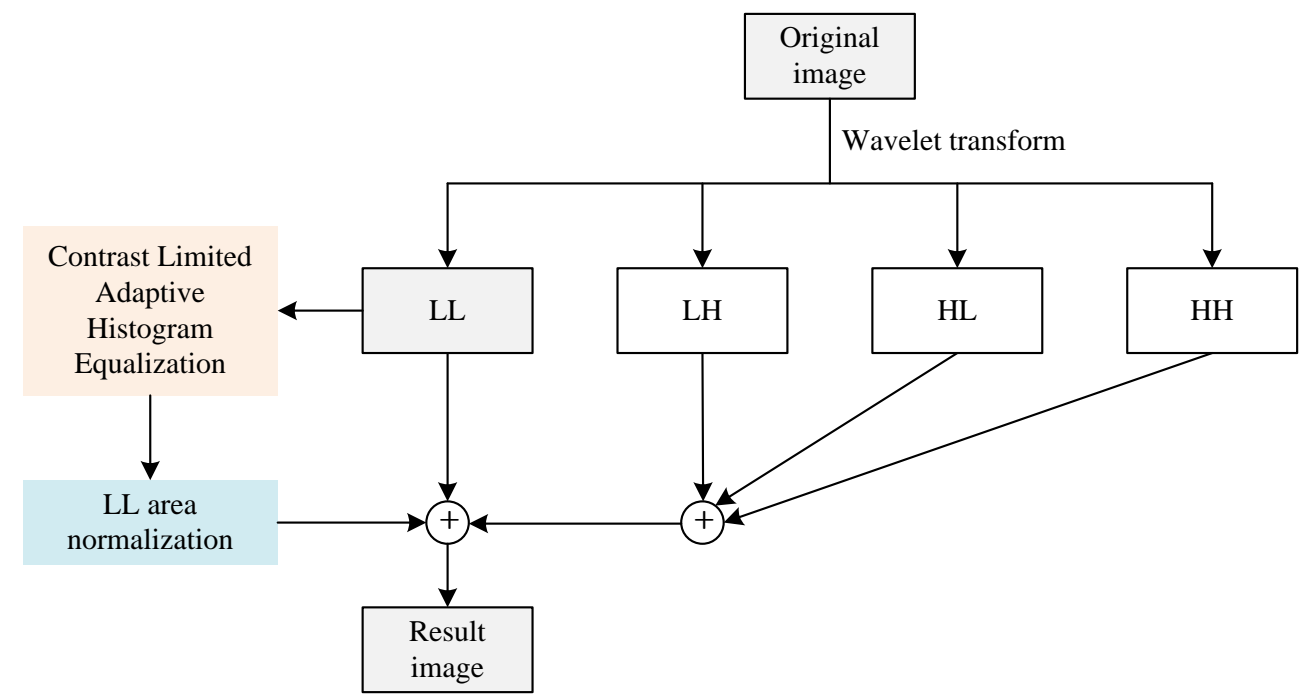

Figure 3. The Flowchart of the Proposed Method.

Once process is finished, to restore the final image, inverse wavelet transform is performed. This process is inverse (or reverse) process of the forward wavelet transform. A pair of 'Haar' wavelet HPF (high pass filter) and LPF (low pass filter) are employed here. This filter pair is called as synthesis process.

\section{Experimental Results and Discussion}

To assess the performance of the proposed algorithm, we provide visual and objective performance. The simulation is conducted using Matlab software with a processor of Intel(R) Core(TM) i5 CPU M460 @2.53GHZ. We used twenty images from LC dataset, which are numbered \#21 to \#40. Figure 4 shows employed test images. The size of test images is either $720 \times 540$ or $540 \times 720$. We used three objective quality evaluation metrics: mean-squared-error (MSE), peak signal-to-noise ratio (PSNR), and structural similarity (SSIM) metrics. The PSNR is calculated as,

$$
P S N R=10 \log _{10}\left(\frac{A^{2}}{M S E}\right)
$$

where MSE describes the mean-squared-error and $A$ represents the maximum possible intensity of the provided image. If a pixel is represented by $b$ bits then $A$ is computed as $2^{b}$-1. Generally speaking, 30 to $50 \mathrm{~dB}$ image is reliable for 8 bits images. For the case of 16 bits images, 60 to $80 \mathrm{~dB}$ is reliable. The MSE is calculated as,

$$
M S E=\frac{1}{m n} \sum_{i=0}^{m} \sum_{j=0}^{n}[\operatorname{original}(i, j)-\operatorname{result}(i, j)]^{2}
$$

where original( $i, j)$ and result( $(i, j)$ stand for original and the result images and $m$ and $n$ are width and height of an image. The third metric is SSIM, which assesses the quality of the result images. If the result image is similar to the original one, SSIM index closes to 1. On the other hand, if the result image is different from the original one, SSIM index closes to 0 . The introduced all three metrics are full reference metrics. 


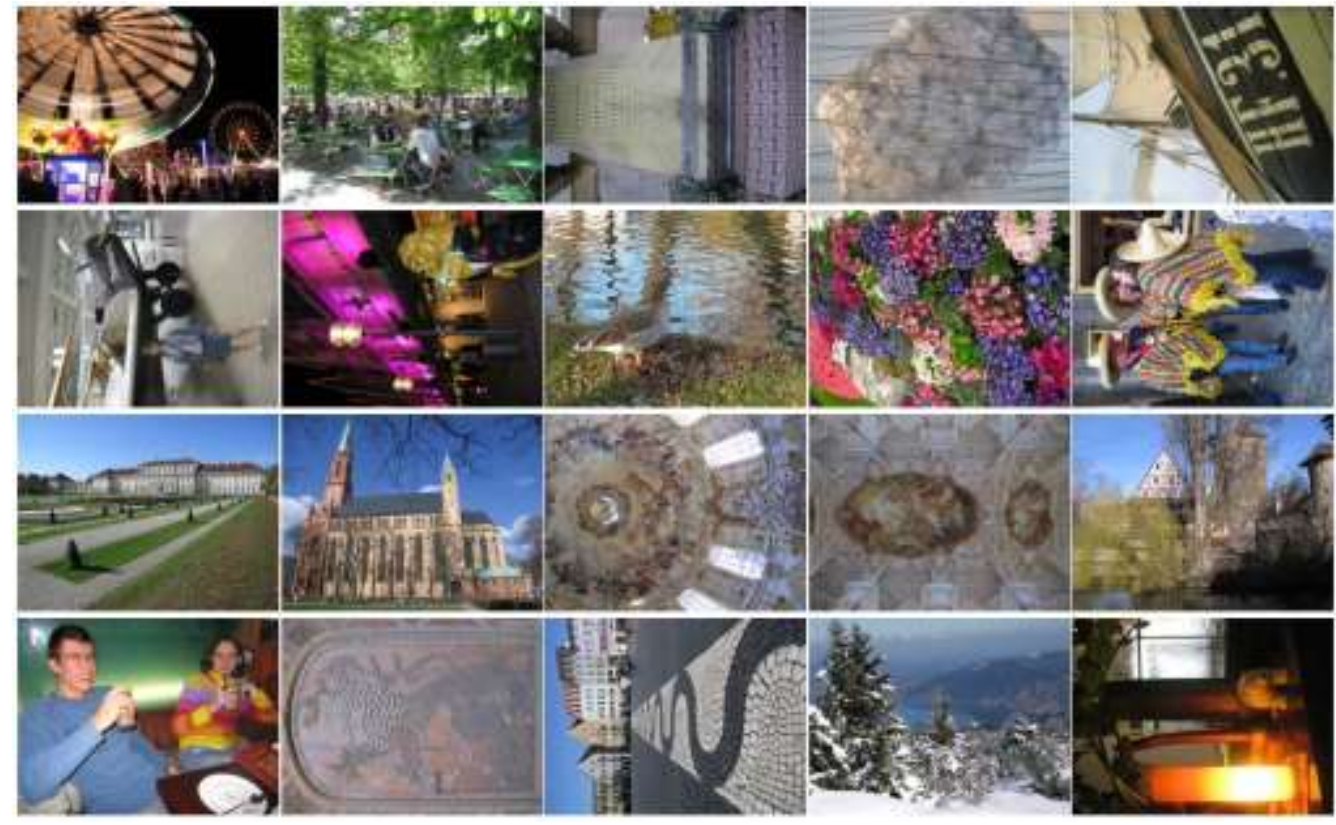

Figure 4. 20 Test LC Images. Image Indices are \#21-\#40

Figures 5 to 8 show several results images. Figures (a) indicate original images. Figures (b) and (c) are result images that were obtained by HE and by the proposed method. Figures (d) and (e) are difference images between 'original and HE' or 'original and the proposed method'. Figures (f) and (g) are SSIM results of HE and the proposed method.

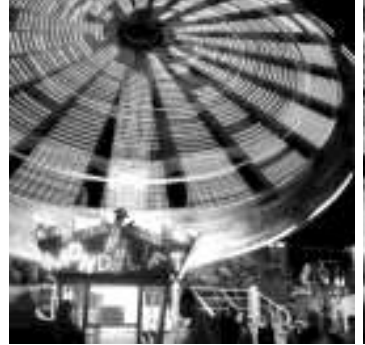

(a)

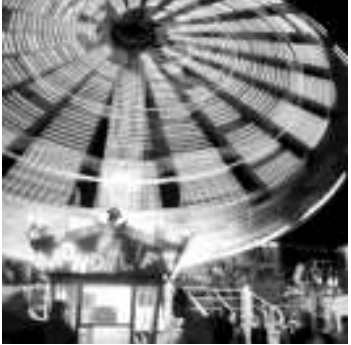

(b)

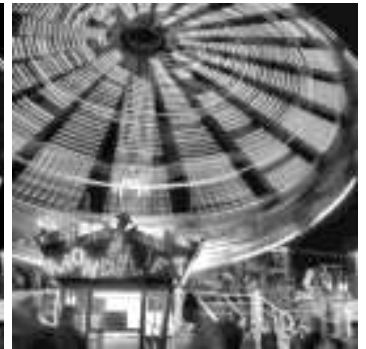

(c)

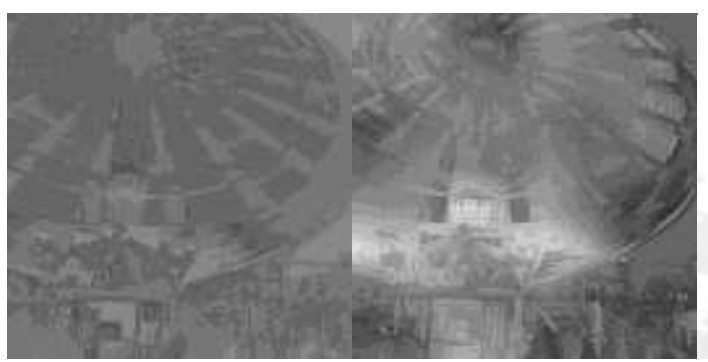

(d)

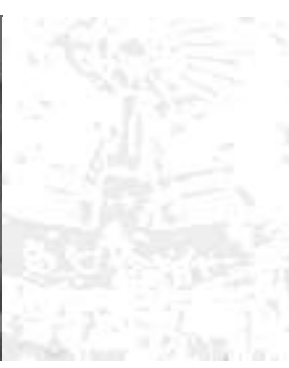

(f)

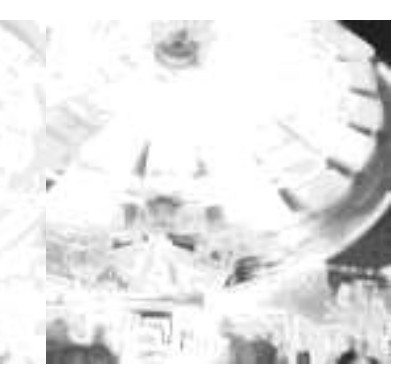

(g)

Figure 5. \#21 LC Test Image. (a) Original Image, (b) HE Result Image, (c) Proposed Method Image, (d) Difference between Original and HE Result, (e) Difference between Original and the Proposed Method, (f) SSIM Map of HE Result, and (g) SSIM Map of the Proposed Method 


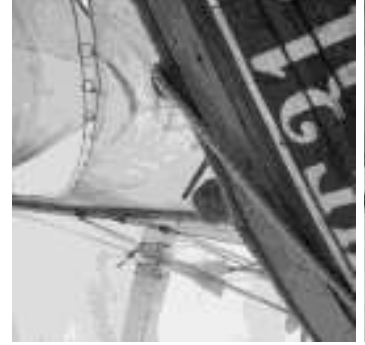

(a)

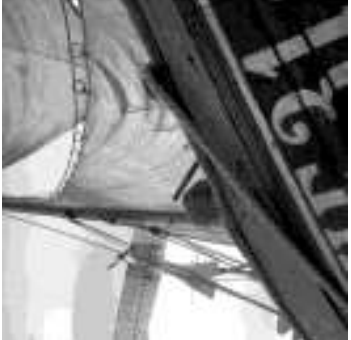

(b)

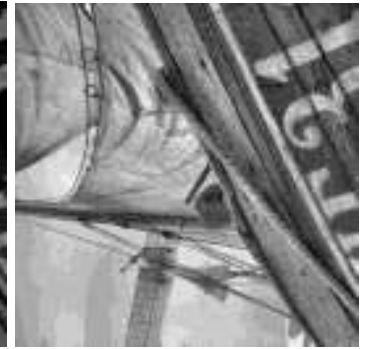

(c)

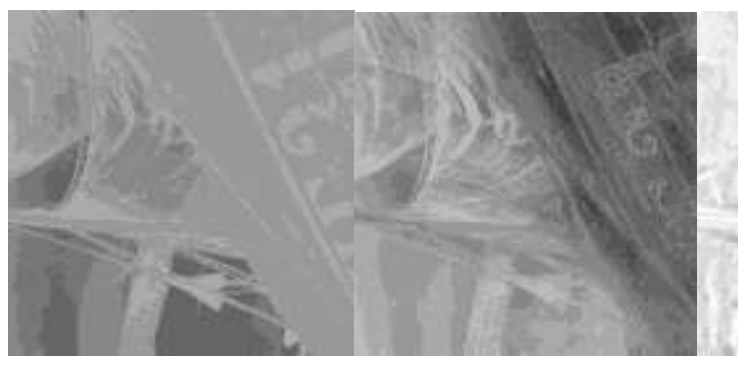

(d) (e)

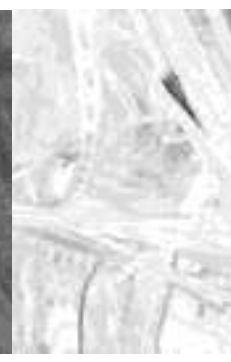

(f)

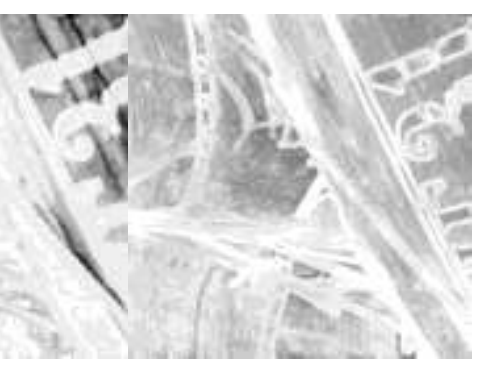

(g)

Figure 6. \#25 LC Test Image. (a) Original Image, (b) HE Result Image, (c) Proposed Method Image, (d) Difference between Original and HE Result, (e) Difference between Original and the Proposed Method, (f) SSIM Map of HE Result, and (g) SSIM Map of the Proposed Method

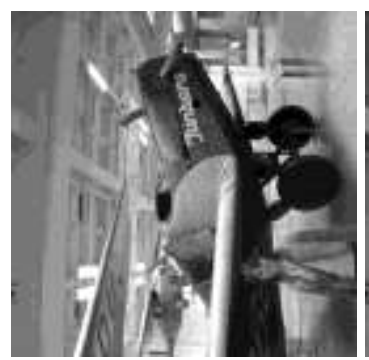

(a)

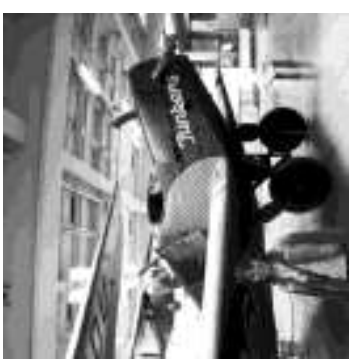

(b)

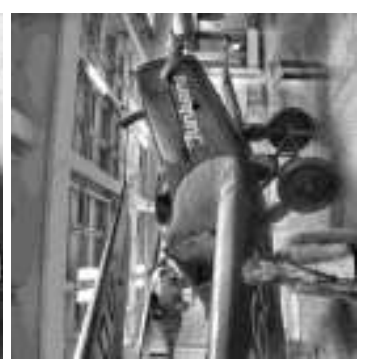

(c)

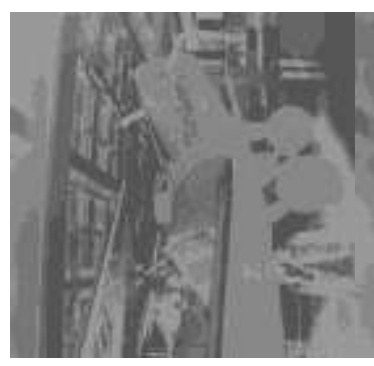

(d)

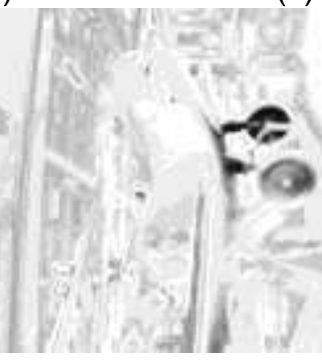

(f)

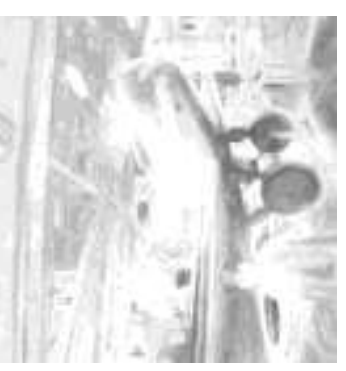

(g)

Figure 7. \#26 LC Test Image. (a) Original Image, (b) HE Result Image, (c) Proposed Method Image, (d) Difference between Original and HE Result, (e) Difference between Original and the Proposed Method, (f) SSIM Map of HE Result, and (g) SSIM Map of the Proposed Method 


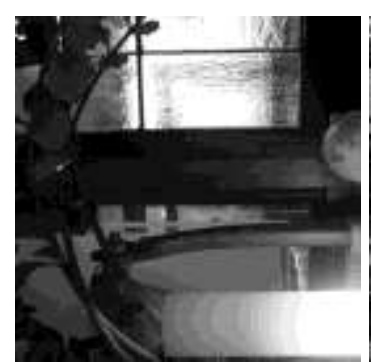

(a)

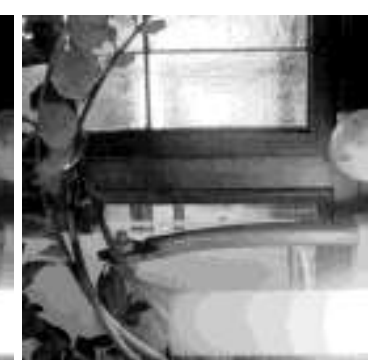

(b)

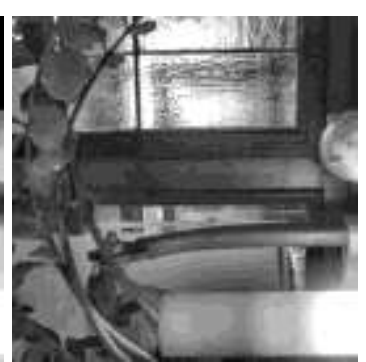

(c)

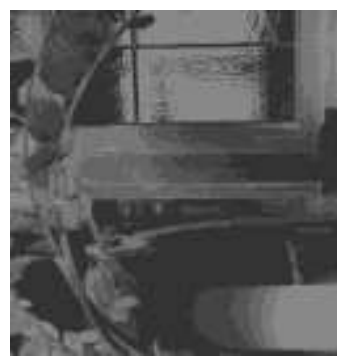

(d)

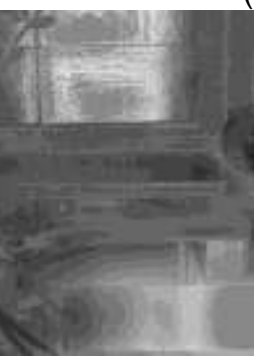

(e)

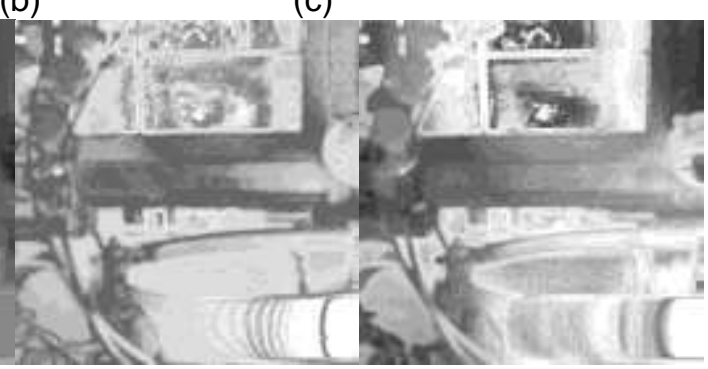

(f)

(g)

Figure 8. \#40 LC Test Image. (a) Original Image, (b) HE Result Image, (c) Proposed Method Image, (d) Difference between Original and HE Result, (e) Difference between Original and the Proposed Method, (f) SSIM Map of HE Result, and (g) SSIM Map of the Proposed Method

Tables 1 and 2 display objective performances of conventional HE and the proposed method. For the proposed method, the average results of MSE, PSNR, and SSIM are 833.3, 19.2, and 0.82678, respectively. Figure 9 shows three figures, which show MSE, PSNR, and SSIM results graphically.

Table 1. Obtained Performance in MSE, PSNR, and SSIM Metrics for Conventional HE Method

\begin{tabular}{|c|r|c|c|c|c|c|c|}
\hline Image \# & MSE & PSNR & SSIM & Image \# & MSE & PSNR & SSIM \\
\hline 1 & 413.97036 & 21.96111 & 0.94951 & 11 & 825.55047 & 18.96337 & 0.85235 \\
\hline 2 & 1002.91030 & 18.11818 & 0.84647 & 12 & 1560.53780 & 16.19806 & 0.76724 \\
\hline 3 & 1101.97340 & 17.70909 & 0.78644 & 13 & 1541.15460 & 16.25234 & 0.68556 \\
\hline 4 & 2642.05840 & 13.91138 & 0.59383 & 14 & 2454.54770 & 14.23109 & 0.58167 \\
\hline 5 & 627.51679 & 20.15455 & 0.86009 & 15 & 1006.72770 & 18.10168 & 0.80411 \\
\hline 6 & 580.23711 & 20.49475 & 0.85035 & 16 & 1033.46260 & 17.98786 & 0.75294 \\
\hline 7 & 4484.65790 & 11.61351 & 0.65935 & 17 & 2678.16560 & 13.85243 & 0.54071 \\
\hline 8 & 579.10267 & 20.50325 & 0.88903 & 18 & 609.80832 & 20.27887 & 0.87605 \\
\hline 9 & 1255.55380 & 17.14245 & 0.85464 & 19 & 377.91882 & 22.35682 & 0.92452 \\
\hline 10 & 422.35103 & 21.87407 & 0.92382 & 20 & 2392.14220 & 14.34293 & 0.67314 \\
\hline & & & & Avg. & 1379.51738 & 17.80239 & 0.78359 \\
\hline
\end{tabular}


Table 2. Obtained Performance in MSE, PSNR, and SSIM Metrics for Proposed Method

\begin{tabular}{|c|c|c|c|c|c|c|c|}
\hline Image \# & MSE & PSNR & SSIM & Image \# & MSE & PSNR & SSIM \\
\hline 1 & 813.20679 & 19.02879 & 0.86525 & 11 & 620.22646 & 20.20530 & 0.86952 \\
\hline 2 & 564.82517 & 20.61166 & 0.90888 & 12 & 557.53839 & 20.66806 & 0.90449 \\
\hline 3 & 686.89604 & 19.76189 & 0.79551 & 13 & 827.39765 & 18.95366 & 0.79358 \\
\hline 4 & 811.94216 & 19.03555 & 0.80025 & 14 & 691.44777 & 19.73321 & 0.75934 \\
\hline 5 & 943.16693 & 18.38492 & 0.81229 & 15 & 735.15742 & 19.46700 & 0.82964 \\
\hline 6 & 599.43184 & 20.35341 & 0.84765 & 16 & 579.39698 & 20.50104 & 0.82117 \\
\hline 7 & 1665.03010 & 15.91658 & 0.67589 & 17 & 451.90800 & 21.58030 & 0.81419 \\
\hline 8 & 1105.03150 & 17.69706 & 0.84464 & 18 & 550.82490 & 20.72067 & 0.88707 \\
\hline 9 & 1246.04570 & 17.17546 & 0.87793 & 19 & 772.57169 & 19.25142 & 0.87280 \\
\hline 10 & 735.20748 & 19.46670 & 0.88461 & 20 & 1709.62890 & 15.80179 & 0.67086 \\
\hline & & & & Avg. & 833.34409 & 19.21572 & 0.82678 \\
\hline
\end{tabular}

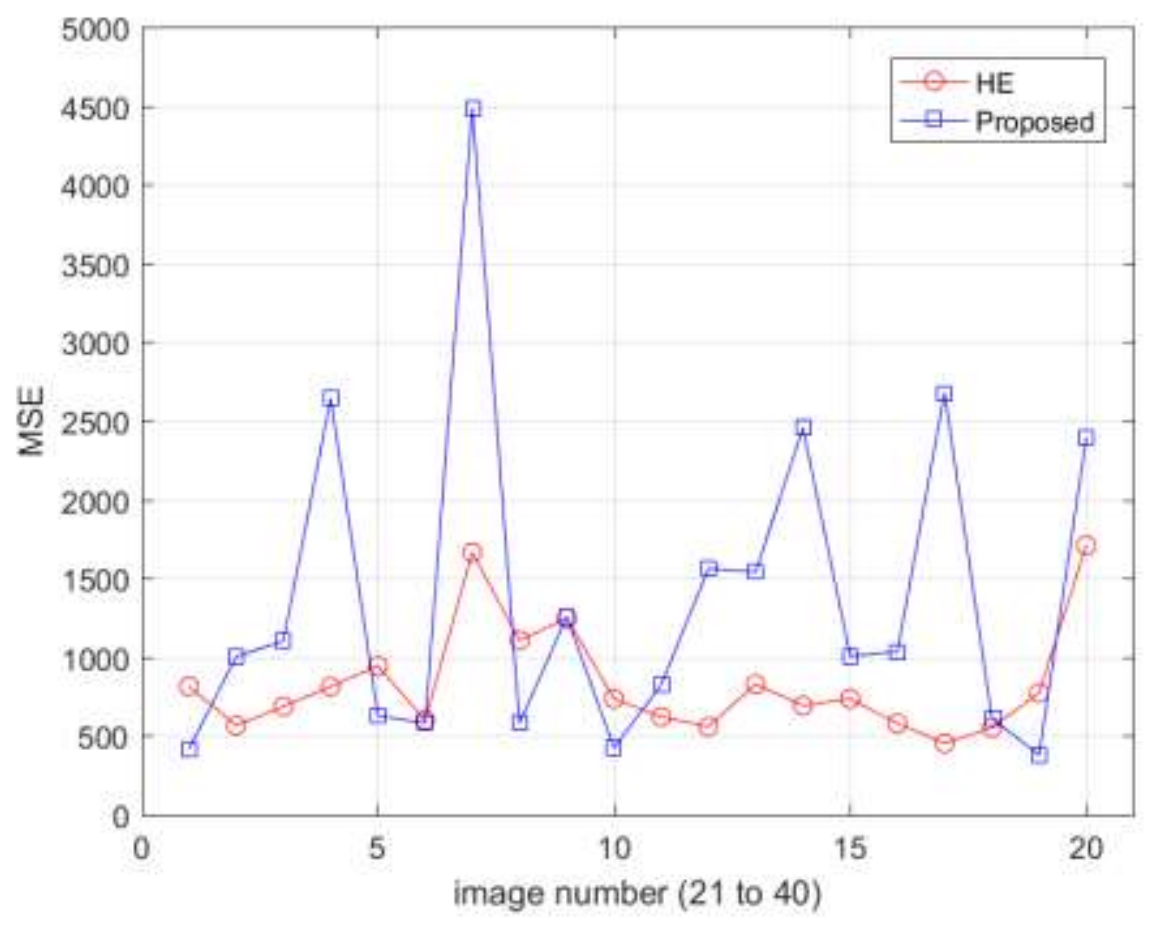

(a) 


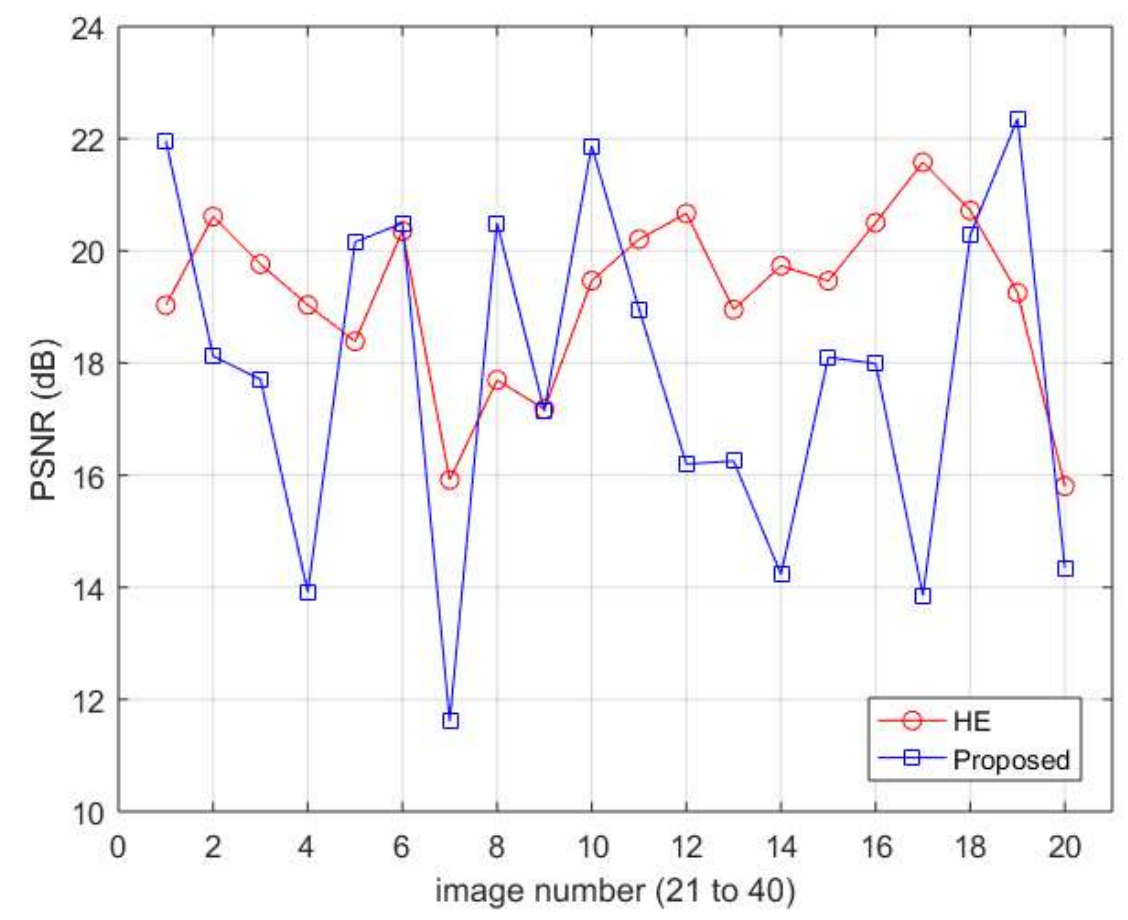

(b)

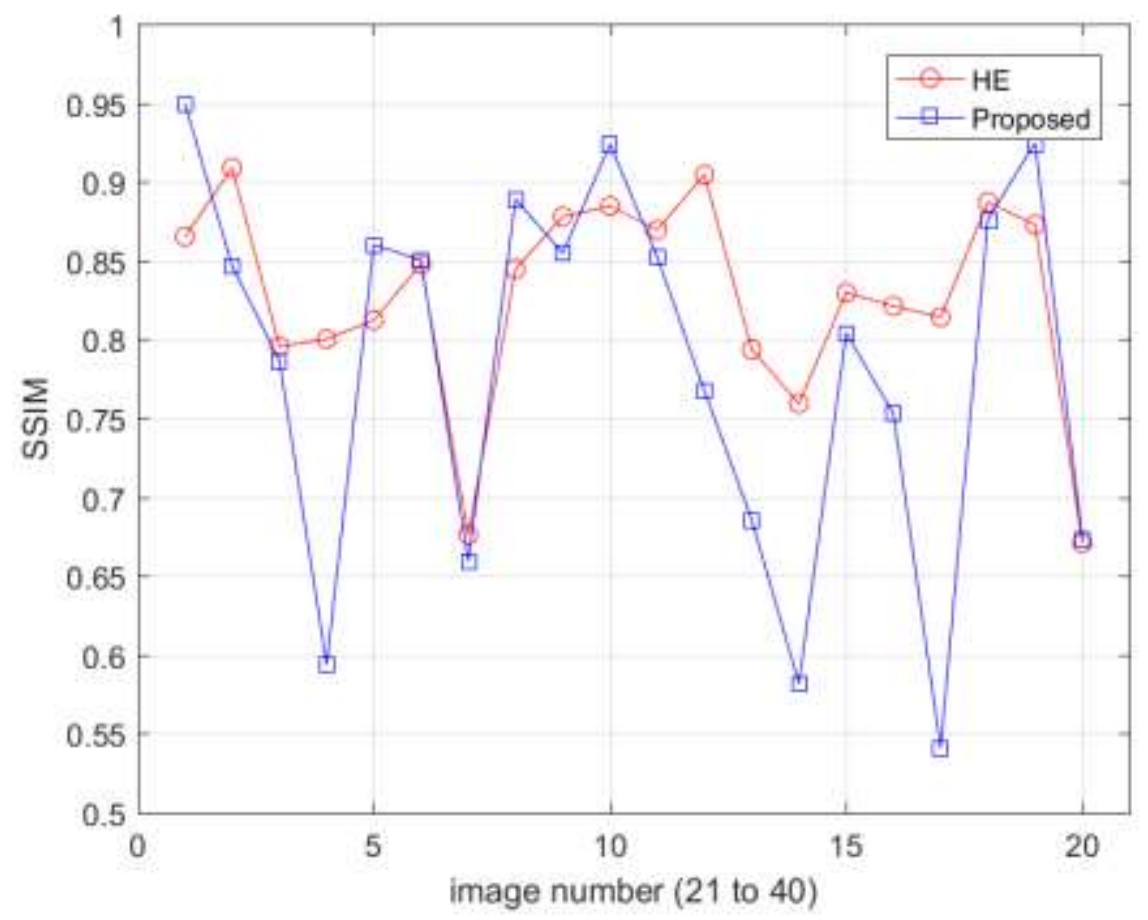

(c)

Figure 9. Performance of the Proposed Method in Three Metrics: (a) MSE, (b) PSNR, and (c) SSIM 


\section{Conclusions}

To improve the quality of result image, we proposed contrast enhancement method. The histogram equalization draws the dynamic range of brightness, and it consequences intensity enhanced image. To enhance contrast of given image, we practiced contrast limited adaptive histogram equalization in low-low band of wavelet transform. Then, we apply low-low band normalization to scale image intensity. Simulation results tells that the presented method outperforms the conventional method.

\section{Acknowledgements}

This work was supported by the National Research Foundation of Korea(NRF) Grant funded by the Korean Government (2015R1D1A1A01058171). This paper is a revised and expanded version of a paper entitled "Contrast Enhancement Method in Wavelet Domain" presented at SIP2016.

\section{References}

[1] S. G. Tanyer, "The cumulative distribution function for a finite data set", in Proc. Signal Processing and Communications Applications Conference (SIU), (2012), pp. 1-3.

[2] J. M. Gauch, "Investigations of image contrast space defined by variations on histogram equalization", CVGIP: Graph. Models Image Process, vol. 54, (1992), pp. 269-280.

[3] J. A. Stark and W. J. Fitzgerald, "An alternative algorithm for adaptive histogram equalization”, Graph. Models Image Process, vol. 58, (1996), pp. 180-185.

[4] R. Hummel, "Image enhancement by histogram transformation", Comp. Graph. Image Process, vol. 6, (1977), pp. 184-195.

[5] Y.-T. Kim, "Contrast enhancement using brightness preserving bi-histogram equalization", IEEE Trans. Consumer Electronics, vol. 43, no. 1, (1997), pp. 1-8.

[6] V. T. Tom and G. J. Wolfe, "Adaptive histogram equalization and its applications", SPIE Applicat. Dig. Image Process. IV, vol. 359, (1982), pp. 204-209.

[7] S. M. Pizer, E. P. Amburn, J. D. Austin, R. Cromartie, A. Geselowitz, T. Greer, B. H. Romeny, J. B. Zimmerman, and K. Zuiderveld, "Adaptive histogram equalization and its variations", Comp. Vis. Graph. Image Process, vol. 39, no. 3, (1987), pp. 355-368.

[8] K. Zuiderveld, "Contrast Limited Adaptive Histogram Equalization", Academic Press Inc., (1994).

[9] X. Chen, G. Jeon, and J. Jeong, "A filter switching interpolation method for deinterlacing”, SPIE Optical Engineering, vol. 51, no. 10, (2012), pp. 107402.

[10] S. J. Park, G. Jeon, and J. Jeong, "Covariance-based adaptive deinterlacing method using edge map", in Proc. IEEE IPTA2010, Paris, France, (2010), pp. 166-171.

[11] S. J. Park, G. Jeon, and J. Jeong, "Deinterlacing algorithm using edge direction from analysis of the DCT coefficient distribution", IEEE Trans. Consumer Electronics, vol. 55, no. 3, (2009), pp. 1674-1681.

[12] G. Jeon, S.J. Park, Y. Fang, M. Anisetti, V. Bellandi, E. Damiani, and J. Jeong, "Specification of efficient block matching scheme for motion estimation in video compression", SPIE Optical Engineering, vol. 48, no. 12, (2009), pp. 127005.

[13] J. Wu, Z. Xu, G. Jeon, X. Zhang, and L. Jiao, "Arithmetic coding for image compression with adaptive weight-context classification", Signal Processing: Image Communication, vol. 28, no. 7, (2013), pp. 727-735.

[14] X. Chen, G. Jeon, and J. Jeong, "Voting-based directional interpolation method and its application to still color image demosaicking”, IEEE Trans. Circuits and Systems for Video Technology, vol. 24, no. 2, (2014), pp. 255-262.

[15] X. Chen, G. Jeon, J. Jeong, and L. He, "Multidirectional weighted interpolation and refinement method for Bayer pattern CFA demosaicking", IEEE Trans. Circuits and Systems for Video Technology, vol. 25, no. 8, (2015), pp. 1271-1282.

[16] J. Wang, G. Jeon, and J. Jeong, "Efficient adaptive de-Interlacing algorithm with awareness of closeness and similarity", SPIE Optical Engineering, vol. 51, no. 1, (2012), pp. 017003. 


\begin{abstract}
Author
Gwanggil Jeon received the BS, MS, and $\mathrm{PhD}$ (summa cum laude) degrees in Department of Electronics and Computer Engineering from Hanyang University, Seoul, Korea, in 2003, 2005, and 2008, respectively. From 2008 to 2009, he was with the Department of Electronics and Computer Engineering, Hanyang University, from 2009 to 2011, he was with the School of Information Technology and Engineering (SITE), University of Ottawa, as a postdoctoral fellow, and from 2011 to 2012, he was with the Graduate School of Science \& Technology, Niigata University, as an assistant professor. $\mathrm{He}$ is currently an associate professor with the Department of Embedded Systems Engineering, Incheon National University, Incheon, Korea. His research interests fall under the umbrella of image processing, particularly image compression, motion estimation, demosaicking, and image enhancement as well as computational intelligence such as fuzzy and rough sets theories. He was the recipient of the IEEE Chester Sall Award in 2007 and the 2008 ETRI Journal Paper Award.
\end{abstract}


International Journal of Signal Processing, Image Processing and Pattern Recognition Vol. 10, No. 7 (2017) 